\title{
Dynamic measurement of the acoustic streaming time constant utilizing an optical tweezer
}

\author{
Christoph Goering $\odot^{*}$ and Jürg Dual $\odot$ \\ ETH Zurich, Institute for Mechanical Systems, Leonhardstr. 21, 8092 Zurich, Switzerland
}

(Received 18 May 2021; accepted 22 July 2021; published 16 August 2021)

\begin{abstract}
The combination of a bulk acoustic wave device and an optical trap allows for studying the buildup time of the respective acoustic forces. In particular, we are interested in the time it takes to build up the acoustic radiation force and acoustic streaming. For that, we measure the trajectory of a spherical particle in an acoustic field over time. The shape of the trajectory is determined by the acoustic radiation force and by acoustic streaming, both acting on different time scales. For that, we utilize the high temporal resolution $(\Delta t=0.8 \mu \mathrm{s})$ of an optical trapping setup. With our experimental parameters the acoustic radiation force on the particle and the acoustic streaming field theoretically have characteristic buildup times of $1.4 \mu \mathrm{s}$ and $1.44 \mathrm{~ms}$, respectively. By choosing a resonance mode and a measurement position where the acoustic radiation force and acoustic streaming induced viscous drag force act in orthogonal directions, we can measure the evolution of these effects separately. Our results show that the particle is accelerated nearly instantaneously by the acoustic radiation force to a constant velocity, whereas the acceleration phase to a constant velocity by the acoustic streaming field takes significantly longer. We find that the acceleration to a constant velocity induced by streaming takes in average about 17500 excitation periods $(\approx 4.4 \mathrm{~ms}$ ) longer to develop than the one induced by the acoustic radiation force. This duration is about four times larger than the so-called momentum diffusion time which is used to estimate the streaming buildup. In addition, this rather large difference in time can explain why a pulsed acoustic excitation can indeed prevent acoustic streaming as it has been shown in some previous experiments.
\end{abstract}

DOI: 10.1103/PhysRevE.104.025104

\section{INTRODUCTION}

In recent years, acoustofluidics has provided many powerful tools. Due to being contactless, label-free, and biocompatible [1-5], acoustofluidic manipulation can be used in medical applications for cancer research [1-4], Alzheimer research [5], targeted drug delivery [6], and for pumping medical fluids [7]. In addition, there are biological [8,9] and engineering applications (e.g., micropumping [7,10-12]).

Most of these applications utilize the acoustic radiation force (ARF) to manipulate objects on the microscale. The ARF is a second-order time-averaged effect that arises from the interaction of an acoustic field scattered at an object surface and a background acoustic field [13-17]. These objects can be solid particles, air bubbles, fluid droplets, and biological samples, as long as their material properties (density $\rho$ and speed of sound $c$ ) are different from the surrounding medium. However, there coexists a fluid motion called acoustic streaming (AS) [18-20]. This motion can arise either from viscous losses in the fluid (Eckhart type streaming [21]) or it can arise in the viscous boundary layer at a fluid to wall interface (Schlichting and Rayleigh streaming [22,23]).

The theoretical derivations usually describe the steady state of the AS field. A theoretical numerical study [24] investigated the temporal buildup of the ARF and AS field. In contrast to the ARF, the viscous drag force arising from AS is independent of the object material properties because it is

\footnotetext{
*goering@imes.mavt.ethz.ch
}

a motion of the fluid. The AS direction coincides with the direction of the relative motion between fluid and particle.

For a spherical object of radius $R$, the drag force in laminar flow scales linearly with the object radius $F_{\mathrm{AS}} \propto R$. In contrast to the $F_{\mathrm{AS}}$, the ARF scales with the volume $F_{\mathrm{ARF}} \propto R^{3}[25]$. Based on the fluid and the object material properties, the $F_{\mathrm{ARF}}$ will dominate over the $F_{\mathrm{AS}}$ if the radius $R$ is greater than the critical radius $R_{\text {crit }}$, where $F_{\mathrm{AS}}=F_{\mathrm{ARF}}$ holds. The direction of $F_{\mathrm{AS}}$ can be different from the $F_{\mathrm{ARF}}$. Therefore, the $F_{\mathrm{AS}}$ is usually undesired.

The ARF and the AS occur not only in the bulk of the fluid, but also on sharp edges of a device [26-29]. So-called microstreaming around the surface of a spherical particle can even cause a sign inversion of the ARF if the viscous boundary layer $\delta$ is sufficiently large [30]. However, there are applications that take advantage of the AS [31-33]: a complete overview of AS applications can be found in [34].

In literature, it is well understood how long it takes until the acoustic field, and hence the ARF, needs to build up [24] and how long the particle focusing takes [25]. However, it is still not fully clear how long it takes for the AS to build up, and what the definition for the analytical AS time constant is. In the acoustofluidics community, it is generally accepted that the buildup for the AS field takes longer than the buildup of the ARF. By using a pulsed actuation of the acoustic field and therefore exploiting this time offset, Hoyos and Castro prevented the buildup of AS $[35,36]$. They varied the number of periods for which the acoustic actuation is switched on and off, respectively. They experimentally showed that for a ratio of about 1 to 1 between 500 on and 
500 off periods the streaming velocity is less than $50 \%$ of its steady-state magnitude, while the ARF is not affected by that much.

Muller and Bruus studied the buildup of the acoustic energy density and streaming velocity with a numerical model [24]. Their model consisted of a fluid cavity without any surrounding structure such as the cavity walls. They found numerically that indeed the ARF builds up significantly faster than the AS. However, the simulations with a pulsed actuation of different ratios of on to off periods did not prevent the buildup of AS because its decay-as the buildup-is slow compared to the ARF. The streaming builds up significantly slower during the on periods; however, it does not decay to its initial value during the off periods. Over time the influence of AS increases because the ARF alternates between some magnitude in the on periods and zero in the off periods. This implies that the simulation of Muller and Bruus could not explain the experimental results by Hoyos and Castro.

In this work, we experimentally measure the time until a $2.06 \mu \mathrm{m}$ spherical silicon-dioxide $\left(\mathrm{SiO}_{2}\right)$ particle moves with constant velocity when accelerated by the ARF and AS. Instead of using a camera, we utilize a data acquisition board (DAQ) with a sampling frequency of $f_{\mathrm{s}}=1.25 \mathrm{MHz}$ to measure the relative particle trajectory as soon as the ultrasound (US) is switched on. This high sampling frequency $f_{\mathrm{s}}$ yields a high temporal resolution of $\Delta t=0.8 \mu \mathrm{s}$. Considering the acoustic excitation frequency $f_{\text {ex }}=4.015 \mathrm{MHz}$, we sample at least every fourth excitation period.

The optical tweezer (OT) for this study has already been successfully applied in the fields of acoustofluidics for stationary force measurements within a microfluidic chip [37,38], as well as acoustic viscous torque investigations [39]. Here, we characterize in a first step the stationary force field in the bulk of the device to ensure that we measure in a second step the time resolved buildup of AS and the ARF separately and not their superposition. The separation is done by choosing a particle position within the acoustic field, where the $F_{\mathrm{AS}}$ and $F_{\mathrm{ARF}}$ are orthogonal to each other. In order to measure in the second step solely the effects of the acoustic field on the particle and not the characteristics of the OT, we alter the usual trapping setup. The modification is that the particle is released from the OT before the acoustic excitation starts and retrapped after it. Hence, during the measurement, just gravity and the forces of the acoustic field act upon the particle. With our modified trapping setup, we are able to measure precisely the ARF and AS induced movement of a single particle in the bulk of the fluid.

Our manuscript is structured as follows. In Sec. II we derive and list all time constants in our system and we compute the traveled distances of a free floating particle in an acoustic field. Those influences need to be considered for our measurement protocol. In addition, we perform numerical AS simulations of our device to further understand the AS field. In Sec. III we explain our experimental setup and its modifications. In Sec. IV we show the results of the stationary force measurement, before explaining our time evolution measurement protocol and the data postprocessing. In Sec. V we show and discuss the results of this study.

\section{PRELIMINARY THEORETICAL CONSIDERATIONS}

\section{A. Time constants}

In our experiments there are multiple time constants that need to be considered. In the center of interest are the evolution of the ARF and the AS field. The acoustic energy $E_{\text {ac }}$, and hence the ARF, has the characteristic time constant [24]

$$
\tau_{\mathrm{ARF}}=\frac{Q}{\omega_{0}}=\frac{Q}{2 \pi f_{\mathrm{ex}}},
$$

with $Q$ being the quality factor of the considered acoustic pressure mode and $f_{\text {ex }}$ the excitation frequency. For the AS field, a theoretical expression for the time constant does not exist. Nevertheless, Muller and Bruus report a momentum diffusion time

$$
\tau_{\mathrm{AS}}=\frac{1}{2 \nu} L^{2}=\frac{\rho_{\mathrm{f}}}{2 \mu_{\mathrm{f}}} L^{2}
$$

as the time constant for the AS field. Here, $L$ is half the radius of a streaming roll, $\nu=\frac{\mu_{\mathrm{f}}}{\rho_{\mathrm{f}}}$ the kinematic viscosity, $\rho_{\mathrm{f}}$ the density, and $\mu_{\mathrm{f}}$ the dynamic viscosity of the fluid. This formula is except for a factor of $\frac{1}{2}$ the same to $\tau_{\text {iner }}$ (Eq. 1.88) in [40], which is the time a Poisseuille flow needs to fully stop in a circular tube of radius $L$ after the immediate removal of its driving pressure. To the best of the authors' knowledge, there is so far no better approximation for the time constant of the AS field.

When a particle is stably trapped, our OT has the properties of a linear mechanical spring [37]. This springlike behavior of the OT has also a time constant until an acting force moves the trapped particle in its equilibrium position. The stiffness of the OT $k_{i}$ is linearly related to a characterization parameter of the OT called the cutoff frequency $f_{\mathrm{c}}=\frac{k_{i}}{2 \pi \gamma}$, with $\gamma$ being Stokes' drag coefficient [37,41]. This frequency is the $-3 \mathrm{~dB}$ point in the Brownian motion power spectrum (more detail in $[37,38])$. We can therefore compute the time constant of the OT as

$$
\tau_{\mathrm{OT}}=\frac{1}{2 \pi f_{c}} .
$$

Lastly, our DAQ system has the time constant $\tau_{\mathrm{QPD}}$, which describes how fast we can measure a sudden change in laser intensity of the OT. This parameter is found by changing the laser intensity at a precise point in time and then extracting the temporal difference until the DAQ measures it.

With the parameters of our experiment (see Table I) the mentioned time constants are as listed in Table II. Hence, with the usual trapping mode of the OT, we cannot measure the ARF and AS because $\tau_{\mathrm{OT}} \approx \tau_{\mathrm{AS}}$ and $\tau_{\mathrm{OT}} \gg \tau_{\mathrm{ARF}}$. In the limit of zero laser power there is no trapping potential and hence $\tau_{\mathrm{ARF}}$ and $\tau_{\mathrm{AS}}$ can be measured.

\section{B. Free particle motion}

If there is no trapping laser power, the spherical particle with mass $m$ will move in the fluid due to some acting force $F$; this force can be gravity, the ARF, the drag force from AS, or a combination of them. The one-dimensional dynamic equation for the particle displacement $q$ far away from any walls is the same for the three spatial directions $\boldsymbol{e}_{x}, \boldsymbol{e}_{y}$, and $\boldsymbol{e}_{z}$,

$$
\ddot{q}=-\frac{F}{m}-\frac{\gamma}{m} \dot{q}=-\tilde{F}-\frac{1}{\tau_{\text {drag }}} \dot{q},
$$


TABLE I. Symbols and physical properties of the fluid, the particle, and the experimental setup. The quality factor $Q$ is extracted from an admittance measurement of the device filled with water and fixed in the microscope as for all measurements. The magnitude of $f_{\mathrm{c}}$ is the usual value in stationary force measurements for the OT.

\begin{tabular}{llll}
\hline \hline Parameter & Symbol & \multicolumn{1}{c}{ Value } & Unit \\
\hline Fluid & & & \\
Density & $\rho_{\mathrm{f}}$ & 1000 & $\mathrm{~kg} \mathrm{~m}^{-3}$ \\
Speed of sound & $c_{\mathrm{f}}$ & 1500 & $\mathrm{~m} \mathrm{~s}^{-1}$ \\
Compressibility & $\kappa_{\mathrm{f}}$ & $4.4 \times 10^{-10}$ & $\mathrm{~Pa}^{-1}$ \\
Dynamic viscosity & $\mu_{\mathrm{f}}$ & 890 & $\mu \mathrm{Pa} \mathrm{s}^{-1}$ \\
Kinematic viscosity & $\nu_{\mathrm{f}}=\frac{\mu_{\mathrm{f}}}{\rho_{\mathrm{f}}}$ & 0.890 & $\mathrm{~mm}^{2} \mathrm{~s}^{-1}$ \\
Particle & & & \\
Density & $\rho_{\mathrm{p}}$ & 1850 & $\mathrm{~kg} \mathrm{~m}^{-3}$ \\
Radius & $R_{2}$ & 1.03 & $\mu \mathrm{m}$ \\
Radius & $R_{4}$ & 2.195 & $\mu \mathrm{m}$ \\
Compressibility & $\kappa_{\mathrm{p}}$ & $1.6 \times 10^{-11}$ & $\mathrm{~Pa}^{-1}$ \\
Device quality factor & $Q$ & 36 & \\
Corner frequency of OT & $f_{\mathrm{c}}$ & $\approx 100$ & $\mathrm{~Hz}$ \\
Excitation frequency & $f_{\mathrm{ex}}$ & 4.015 & $\mathrm{MHz}$ \\
\hline \hline
\end{tabular}

with $F$ being a force acting along the direction of $q$ and

$$
\tau_{\text {drag }}=\frac{m}{\gamma}=\frac{V \rho_{\mathrm{p}}}{6 \pi R \mu_{\mathrm{f}}}=\frac{2}{9} R^{2} \frac{\rho_{\mathrm{p}}}{\mu_{\mathrm{f}}} .
$$

Here, $R$ is the particle radius, $V$ the particle volume, and $\rho_{\mathrm{p}}$ the particle density. In microfluidics the viscous effects dominate over the inertial effects [40]. Therefore, we neglect $\ddot{q}$ for further calculations. Solving the modified first order ordinary differential equation

$$
\dot{q}=-\tau_{\text {drag }} \tilde{F}=-\frac{F}{m} \tau_{\text {drag }},
$$

with the initial condition $\left.q\right|_{t=0}=0$, gives the linear relation $q(t)=-\tau_{\text {drag }} \tilde{F} t$ with the integration constant being zero.

As already mentioned, we measure while there is no trapping potential of the OT. Therefore, only gravity and the forces from the acoustic field act on the particle. In our experiment we have for $F$ along $\boldsymbol{e}_{y}$ a spatially varying force with a maximal value of $0.5 \mathrm{pN}$ and along $\boldsymbol{e}_{z}$ the buoyancy corrected gravitational force $\tilde{m} g=V\left(\rho_{\mathrm{p}}-\rho_{\mathrm{f}}\right) g$ with a magnitude of $38.2 \mathrm{fN}$ and an acoustic force with a maximal value of $0.25 \mathrm{pN}$

TABLE II. Overview of time constants $\tau_{\mathrm{i}}$ for the system. The values are obtained by using the values from Table I and Eqs. (1) to (3) and (5). $\tau_{\mathrm{QPD}}$ is measured, $\Delta t_{\mathrm{DAQ}}=\frac{1}{f_{\mathrm{s}}}$, and $t_{0}=\frac{1}{f_{\mathrm{ex}}}$.

\begin{tabular}{llr}
\hline \hline Symbol & $\tau_{i}(\mathrm{~ms})$ & $\frac{\tau_{i}}{t_{0}}(-)$ \\
\hline$\tau_{\mathrm{OT}}$ & 1.59 & 6383.9 \\
$\tau_{\mathrm{QPD}}$ & 0.050 & 200.8 \\
$\tau_{\mathrm{ARF}}$ & 0.0014 & 5.6 \\
$\left.\tau_{\mathrm{AS}}\right|_{L=\frac{H}{2}}$ & 1.44 & 5781.6 \\
$\left.\tau_{\mathrm{AS}}\right|_{L=\frac{H}{4}}$ & 0.35 & 1405.3 \\
$\tau_{\mathrm{drag}}$ & 0.00049 & 2.0 \\
$\Delta t_{\mathrm{DAQ}}$ & 0.0008 & 3.2 \\
\hline \hline
\end{tabular}

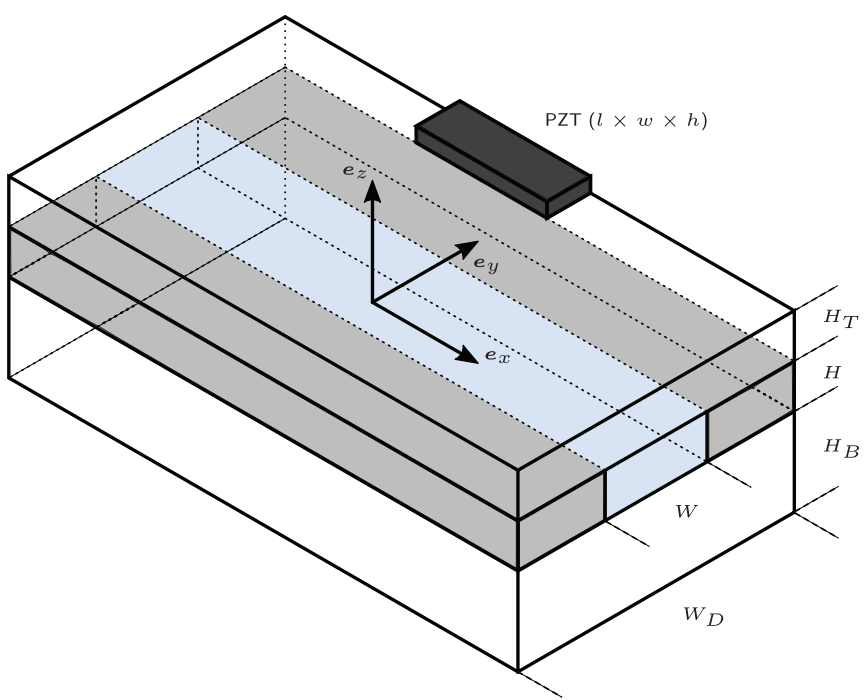

FIG. 1. Sketch of device. The light-gray area is the silicon channel walls, the light-blue area is the fluid cavity, and the dark-gray block is the piezoelectric element. The total length of the device is $76 \mathrm{~mm}$. All dimensions are as listed in Table III.

(for acoustic force magnitudes, see Fig. 6). As we will explain later (see Sec. IV), we have $25 \mathrm{~ms}$ without any laser power where the particle will solely move due to gravity and then 30 ms of US excitation where also the acoustic forces are acting.

Hence a spherical $\mathrm{SiO}_{2} R_{2}=1.03 \mu \mathrm{m}$ particle will have moved $0 R_{2}$ along $\boldsymbol{e}_{y}$ and $0.05 R_{2}$ along $\boldsymbol{e}_{z}$ after $25 \mathrm{~ms}$ with just gravity acting. And, after $55 \mathrm{~ms}$, when there are additionally constant acoustic forces, the particle will have traveled distances of $0.84 R_{2}$ and $0.54 R_{2}$ along $\boldsymbol{e}_{y}$ and $\boldsymbol{e}_{z}$, respectively. For the latter, $0.54 R_{2}$ is the sum of $0.12 R_{2}$ due to gravity and $0.42 R_{2}$ due to the force from the acoustic field.

\section{Numerical streaming simulations}

To understand the influences and implications of the AS on our measurements, we simulate with COMSOL Multiphysics 5.6 (COMSOL Inc., Stockholm, Sweden) two two-dimensional structures that relate to the experimental device-one with just the fluid cavity as the baseline model (cavity-only model) and the other with added structure around the cavity to reflect our real device (whole-device model). See the Supplemental Material [42] for both models as one .mph file. For both we follow the work of Muller and Bruus [24] in terms of the fluid mesh size.

We model a two-dimensional $y z$ slice of the whole device as seen in Fig. 1 without the piezoelectric transducer (PZT) and its glue layer. Therefore, this whole-device model consists of two glass, two silicon, and one water domain. We utilize the Solid Mechanics (solid) interface for the silicon and glass. For the cavity we employ the Creeping Flow (spf)

TABLE III. Overview of device dimensions.

\begin{tabular}{lcccccccc}
\hline \hline Symbol & $W$ & $H$ & $W_{D}$ & $H_{T}$ & $H_{B}$ & $l$ & $w$ & $h$ \\
\hline Value $(\mathrm{mm})$ & 3 & 0.1 & 26 & 0.13 & 0.9 & 20 & 4 & 0.5 \\
\hline
\end{tabular}


(a)

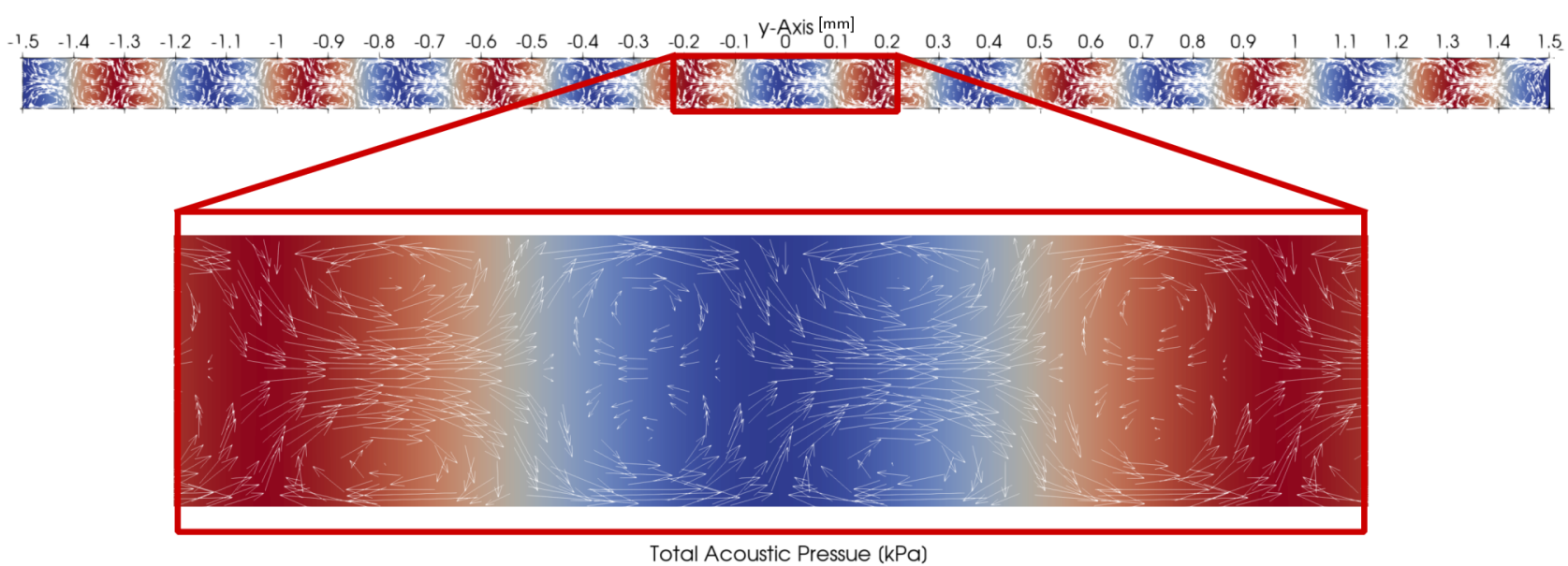

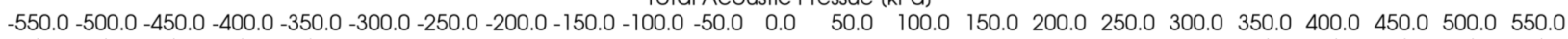

(b)

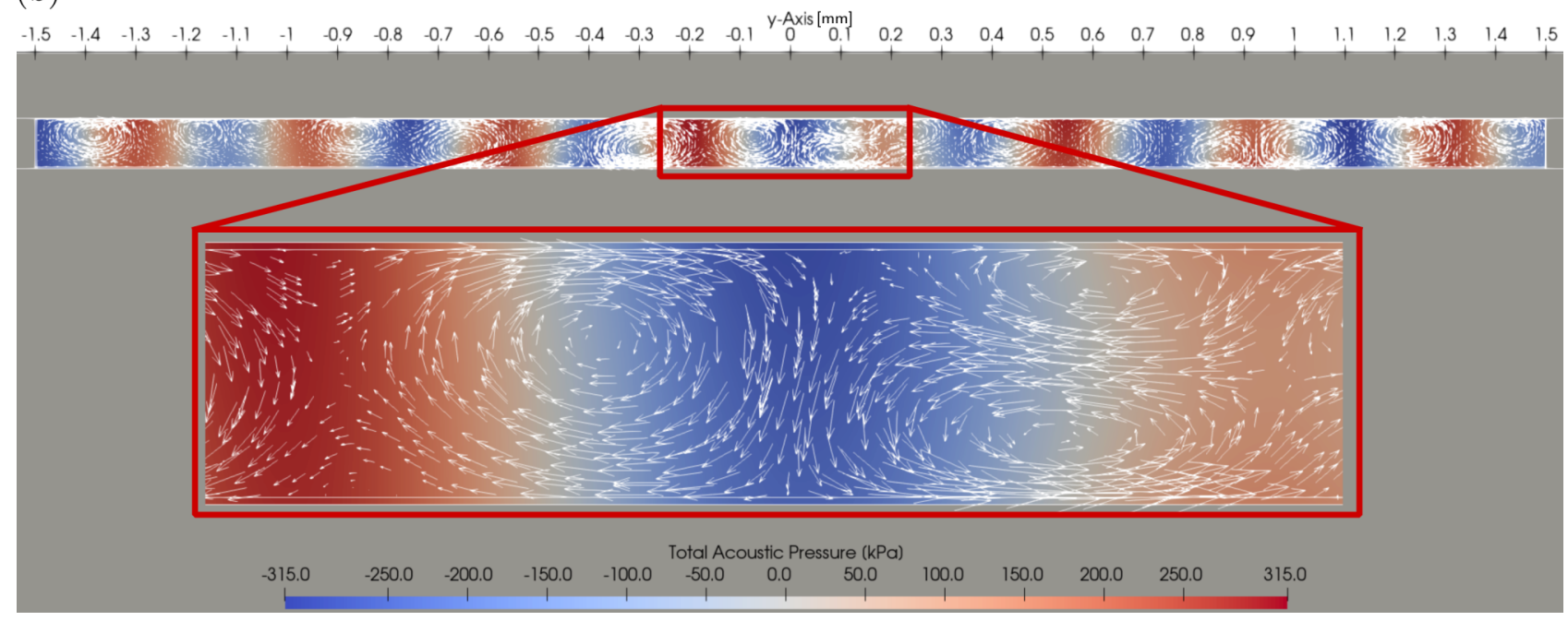

FIG. 2. Results for streaming simulations of a cavity-only model (a) and a model with surrounding structure (b). The color map shows the total acoustic pressure and the white arrows the streaming flow. For both simulations $f_{\max }$ is the frequency of maximal acoustic energy density $E_{\text {ac }}$ for a pressure mode with 16 nodal lines. The pressure mode for both simulations is the same besides the phase shift of $\pi$. (a) Streaming simulation for cavity-only model at $f_{\max }=3.987 \mathrm{MHz}$. (b) Streaming simulation for whole-device model at $f_{\max }=3.745 \mathrm{MHz}$. The gray area is the structure around the cavity.

interface with the spatial variation of the Reynolds Stress as source and the Stokes drift as the boundary condition. Also in the cavity, we use the Thermoviscous Acoustics (ta) interface. Lastly, we couple the Solid Mechanics with the Thermoviscous Acoustics via Thermoviscous Acousticstructure Boundary ( $\mathrm{tsb}$ ). The cavity-only model solely needs the Creeping Flow and the Thermoviscous Acoustics interface without multiphysics coupling.

Besides the added structure around the cavity, the main difference between the two models is the location of the excitation. The whole-device model has as boundary condition a prescribed displacement along $\boldsymbol{e}_{z}$ of $z_{\mathrm{BD}}=0.1 \mathrm{~nm}$, where the PZT is glued onto the device. The cavity-only model, however, has a prescribed constant velocity of its left cavity wall along $\boldsymbol{e}_{y}$ of $\dot{y}_{\mathrm{BD}}=25 \mathrm{~mm} \mathrm{~s}^{-1}$. This magnitude corresponds to the mean wall velocity of the whole-device model, where the excitation is at the PZT. With those two boundary conditions the acoustic pressure is $310 \mathrm{kPa}$ for the whole model and $550 \mathrm{kPa}$ for the cavity-only model at their respective 16 nodal pressure line frequency with maximal acoustic energy density $E_{\text {ac }}$. The discrepancy in pressure amplitude comes from the applied boundary conditions of respective models.

The respective frequencies of maximal $E_{\mathrm{ac}}(3.9876 \mathrm{MHz}$ and $3.7450 \mathrm{MHz}$ ) inside the cavity while having a 16 nodal line mode were determined with a frequency sweep. This is the same mode we have in our experiment as well. For the streaming simulation, we employ a stationary study of the Creeping Flow interface that uses the results from the frequency domain study in its source term and as the boundary conditions.

Figure 2 shows the results for the pressure and streaming fields of both models. The magnifications correspond to the 
area where we perform our measurements in the experiment. One can see that the simulated pressure fields are qualitatively the same; however, the streaming fields differ to a great extent. The cavity-only simulation depicts spatially repetitive streaming rolls over the whole fluid domain. In the bulk of the fluid is Rayleigh streaming. However, near-boundary Schlichting streaming is not visible because the viscous boundary layer is relatively small. In contrast to that, the simulation for the whole-device model has a nonspatially repetitive streaming field. There are regions where its similar to the cavity-only model streaming field. But, the streaming pattern is nonrepetitive and exhibits strong local differences. As a consequence, care must be taken to choose a measuring point where the $F_{\mathrm{AS}}$ and $F_{\mathrm{ARF}}$ are orthogonal to each other to ensure no superposition of forces.

Although the clamping of the microscope setup and the oil immersion layer for the lens are excluded in the model with structure, one can see that the streaming field is a local, nonperiodic effect, whereas the pressure field is spatially periodic. We expect that these tendencies remain the same when the clamping, the immersion layer, and the PZT are added.

\section{EXPERIMENTAL SETUP}

\section{A. Optical trap setup}

Our OT has already been applied in several other publications $[37-39,41]$ to the field of ARF and AS measurements in bulk acoustic wave (BAW) devices. All components are described there extensively. We highlight here the position detection system and the modifications from the force measurement setup that were necessary for this study. These modifications are needed because we use one $785 \mathrm{~nm}$ near-infrared diode laser (LuxX 785-200, Omicron Laser, Rodgau-Dudenhofen, Germany) for the optical trapping and also for the optical position detection. We detect the position of the trapped particle relative to the trap center by monitoring the voltages of two quadrant photodiodes (QPD) placed in the back focal plane (see Fig. 3). It is possible to resolve the movement of the particle in all three dimensions. However, the in-plane $(x y)$ and the axial $(z)$ position detection differ.

For the $x y$ position the laser beam is focused onto the QPDxy (see also Fig. 3) such that the spot diameter is about

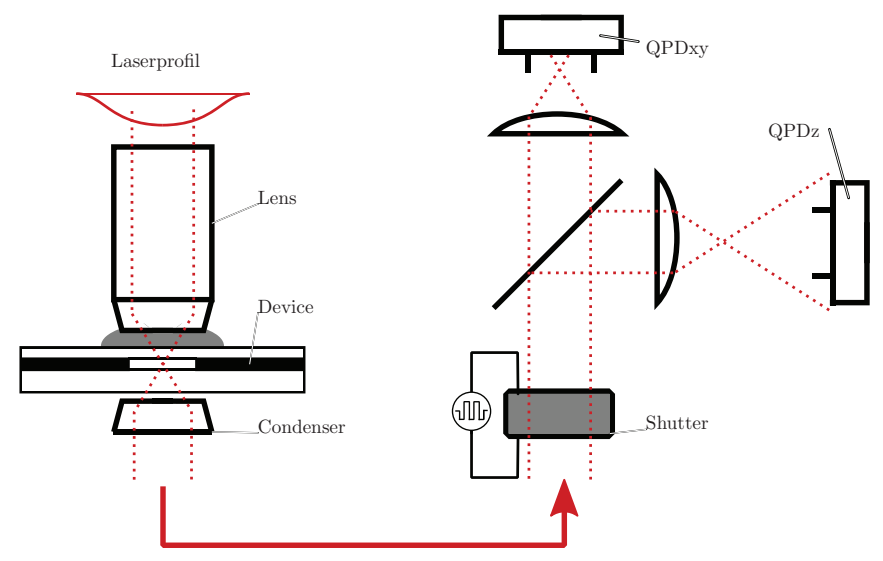

FIG. 3. Shutter location in laser path and schematic of laser path through microscope setup; full details on the setup in $[37,41]$. five times smaller than the opening aperture of the QPDxy [41]. An in-plane movement of the trapped particle changes the spot location on the QPDxy, which results in a voltage change on the four quadrants. As long as the spot is within the QPD opening, the spatial movement is linearly related to the QPD voltage. By summing and subtracting these four voltages from each other, one can get the values corresponding to a movement along $\boldsymbol{e}_{x}$ and $\boldsymbol{e}_{y}$ separately [41].

For the axial $z$ position a second QPD is needed and this QPD is overfilled with the laser spot (see also Fig. 3). When the particle moves axially the spot diameter changes its size. If the diameter decreases more intensity is measured by QPDz and leads to a higher voltage and vice versa. For small movements $(\Delta z<R)$ the relation is linear [43].

When converting the measured voltage changes from QPDxy and QPDz to the particle displacement with unit of meters, the $x y$ voltage and the $z$ voltage have different scaling. The three voltage-meter conversion factors are found by calibrating the OT via the power spectrum analysis of the trapped particle Brownian motion [37-39].

As discussed before, the time constant $\tau_{\mathrm{OT}}$ of the OT is larger than the time constant for the ARF and AS (see Table II). Therefore, we need to switch the laser off and then monitor the particle trajectory without the trapping forces, in order to measure the time evolution of the particle movement and not the time constant of the optical trap. This means the particle is not stably trapped while measuring. However, we need the laser light for the position detection on the QPDs. Therefore, we reduce the laser power to a minimum such that the resulting trapping forces are negligibly small. As a result of the low power, the voltage magnitude decreases significantly on the QPDs, such that it is not measurable anymore. Thus we exchange the neutral density (ND) filters from the force measurement setup [37,39] with the fast optical shutter FOS-NIR(1100) (LC TEC, Borlänge, Sweden). This filter is specified to open from $0 \%$ to $90 \%$ transmittance in less than $15 \mathrm{~ms}$ and close from $100 \%$ transmittance to $10 \%$ in less than $5 \mathrm{~ms}$. The ND filters and the shutter are needed to reduce the intensity on the QPDs and prevent overexposure and hence damage. The transmittance of the shutter can be controlled with the applied driving voltage. Before and after the measurements the shutter is almost completely closed to mimic the ND filters and opened for the actual measurement with reduced laser power.

Lastly, we operate the laser in the so-called analog modulation mode such that the output laser power is proportional to an externally applied dc voltage which is sampled with more than $1.5 \mathrm{MHz}$ by the laser controller unit. The low power mode for the position detection is operated with less than $0.5 \mathrm{~mW}$. The low voltage dc signal for the laser averaged $84.13 \mathrm{mV}$ with a standard deviation of $0.13 \mathrm{mV}$ provides a very consistent voltage and hence laser power. With this power the trapping potential is too weak to keep the particle inside the focus of the laser beam in any of the three spatial directions. The usual laser power for the stationary force measurements is between $100 \mathrm{~mW}$ and $175 \mathrm{~mW}$.

\section{B. Controller timing and data acquisition}

The data acquisition (DAQ) board NI-USB 6356 (National Instruments, Austin, TX, USA), the laser power, the 


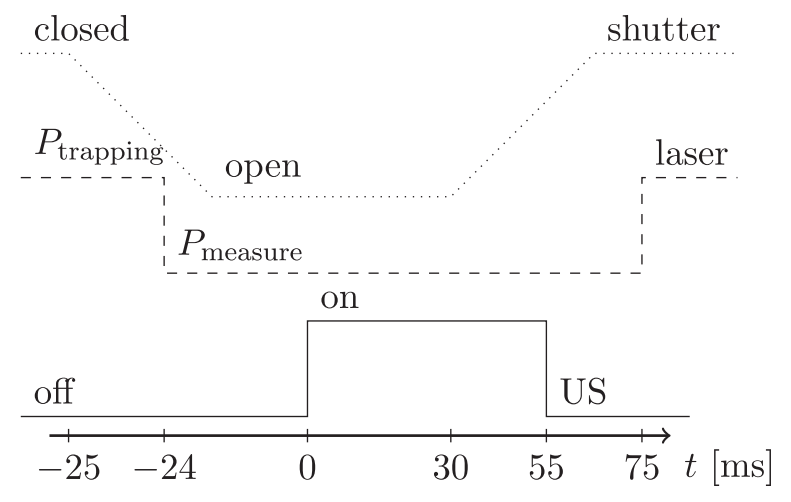

FIG. 4. Schematic of controller timings for the shutter, the laser, and the US. During the $P_{\text {measure }}$ state the particle is not trapped by the OT. In the time interval ( $0 \mathrm{~ms}, 30 \mathrm{~ms})$ (1) the shutter is fully opened, (2) the US is switched on, and (3) the particle is free to move. During this interval the measurement is performed.

piezoexcitation voltage, and the shutter transmittance are actuated in a defined sequence. We use an Arduino Board with two 12-bit DAC units (MCP4725, Adafruit, New York, NY, USA) for controlling the timing and the dc voltage for the laser. The timings are depicted in Fig. 4. For $t<-24 \mathrm{~ms}$ the laser is in its high power state and keeps the particle fixed in position against external forces. At $t=-25 \mathrm{~ms}$ the shutter starts opening. The opening time is specified with less than 15 $\mathrm{ms}$ from $0 \%$ transmittance to $90 \%$. At $t=-24 \mathrm{~ms}$ the laser power changes to its low power state. Hence the particle is free to move and starts its sedimentation. At $t=0 \mathrm{~ms}$ the US is switched on. For $30 \mathrm{~ms}$ the shutter is fully opened, the particle is free to move, and the US is on. Then the shutter starts to close again. In these $30 \mathrm{~ms}$ we measure the time evolution of the particle. At $t=55 \mathrm{~ms}$ the US is switched off and at $t=75 \mathrm{~ms}$ the laser power is increased to its high power state. The time between two consecutive measurements is greater than $2 \mathrm{~s}$, such that the fluid within the cavity is fully at rest again.

\section{Device, particles, and fluid}

Our device is a glass-silicon-glass device manufactured by Gesim GmbH (Radeberg, Germany). The material of the two glasses is B33 from Schott (Mainz, Germany). A sketch is shown in Fig. 1 and its dimensions are listed in Table III. The top glass and the fluid cavity are limited in the $\boldsymbol{e}_{z}$ direction because our microscope setup cannot focus deeper than $250 \mu \mathrm{m}$ $[37,41]$. We define the origin of our coordinate system so that $z=0$ is in the middle of the fluid cavity and $y=0$ is in the middle between the silicon cavity walls. We use as a reference point $x=0$ such that it is approximately in the middle of the PZT length $l$. For all reported measurements we use the same position $x_{\text {ref }}$ as reference for $x=0$.

The fluid cavity is in the middle between the two silicon layers and the PZT is a PZ 26 element from Meggit A/S (Kvistgaard, Denmark). It is glued with Epo-Tek (Billerica, MA, USA) H20S two component epoxy onto the device. It is located at the edge of the device in $\boldsymbol{e}_{y}$ direction and centered along the long side. The small height of the PZT is necessary to prevent physical contact with the microscope lens.
Our particles are silicon-dioxide $\left(\mathrm{SiO}_{2}\right)$ particles from (microParticles $\mathrm{GmbH}$, Berlin, Germany) with a diameter of $D_{2}=2.06 \mu \mathrm{m}$. For the device characterization we also use particles from the same manufacturer with the same material properties, but with a diameter of $D_{4}=4.39 \mu \mathrm{m}$. The particles are immersed in filtered $(0.2 \mu \mathrm{m})$ and distilled water. To avoid particle-particle interactions during the experiment, we keep the particle concentration low.

We use the $2.06 \mu \mathrm{m}$ particles because they are the smallest particles that work well in our OT. In addition, the critical radius where the ARF equals the drag force from AS can be found via [44]

$$
R_{\text {crit }}=\sqrt{\frac{3}{\Phi}} \delta,
$$

where $\Phi$ is the acoustic contrast factor with thermoviscous correction [45]

$$
\begin{aligned}
\Phi(\tilde{\kappa}, \tilde{\rho}, \tilde{\delta}) & =\frac{1}{3} f_{1}(\tilde{\kappa})+\frac{1}{2} \operatorname{Re}\left[f_{2}(\tilde{\rho}, \tilde{\delta})\right], \\
f_{1}(\tilde{\kappa}) & =1-\tilde{\kappa}, \quad \tilde{\kappa}=\frac{\kappa_{\mathrm{p}}}{\kappa_{\mathrm{f}}}, \\
f_{2}(\tilde{\rho}, \tilde{\delta}) & =\frac{2[1-\Gamma(\tilde{\delta})](\tilde{\rho}-1)}{2 \tilde{\rho}+1-3 \Gamma(\tilde{\delta})}, \quad \tilde{\rho}=\frac{\rho_{\mathrm{p}}}{\rho_{\mathrm{f}}}, \\
\Gamma(\tilde{\delta}) & =-\frac{3}{2}[1+\mathrm{i}(1+\tilde{\delta})] \tilde{\delta}, \\
\tilde{\delta} & =\frac{\delta}{R}, \quad \delta=\sqrt{\frac{\mu_{\mathrm{f}}}{\rho_{\mathrm{f}} \pi f}} .
\end{aligned}
$$

Here $\kappa_{\mathrm{p}}$ is the particle and $\kappa_{\mathrm{f}}$ the fluid compressibility, $\delta$ the viscous boundary layer thickness, and $\mathrm{i}$ the imaginary unit. For our parameters (see Table I) $R_{\text {crit }}$ is equal to $0.63 \mu \mathrm{m}$ and $0.65 \mu \mathrm{m}$, with and without $(\tilde{\delta}=0)$ thermoviscous correction, respectively.

With increasing particle size, two effects take place: (1) the ratio between $\mathrm{ARF}\left(\propto R^{3}\right)$ and $\mathrm{AS}\left(F_{\mathrm{AS}} \propto R\right)$ magnitude increases, because of their respective scaling, and (2) the measurement time decreases, because a greater ARF leads to more displacement, which in turn makes retrapping more difficult.

\section{EXPERIMENTAL PROCEDURE}

\section{A. Stationary force measurement}

In preparation for the time evolution measurement, where a spatial position of orthogonal AS forces and ARFs is beneficial, we characterized our device with two sets of stationary force measurements at a constant excitation frequency. For those measurements the optical trapping force is greater than the acoustic forces. One measurement was with a $2.06 \mu \mathrm{m}$ and the other with a $4.39 \mu \mathrm{m}$ diameter particle. For changing the particle size we needed to empty and refill the device. We kept the ambient conditions and experiment settings between the two measurements as constant as possible. For the measurements with the $2.06 \mu \mathrm{m}$ particle the ambient temperature was $24.49^{\circ} \mathrm{C}$ in average with a standard deviation of $0.10^{\circ} \mathrm{C}$ and for the measurement with the $4.39 \mu \mathrm{m}$ particle the average temperature was $24.78^{\circ} \mathrm{C}$ with a standard deviation of $0.25^{\circ} \mathrm{C}$ ensuring the same experimental conditions 

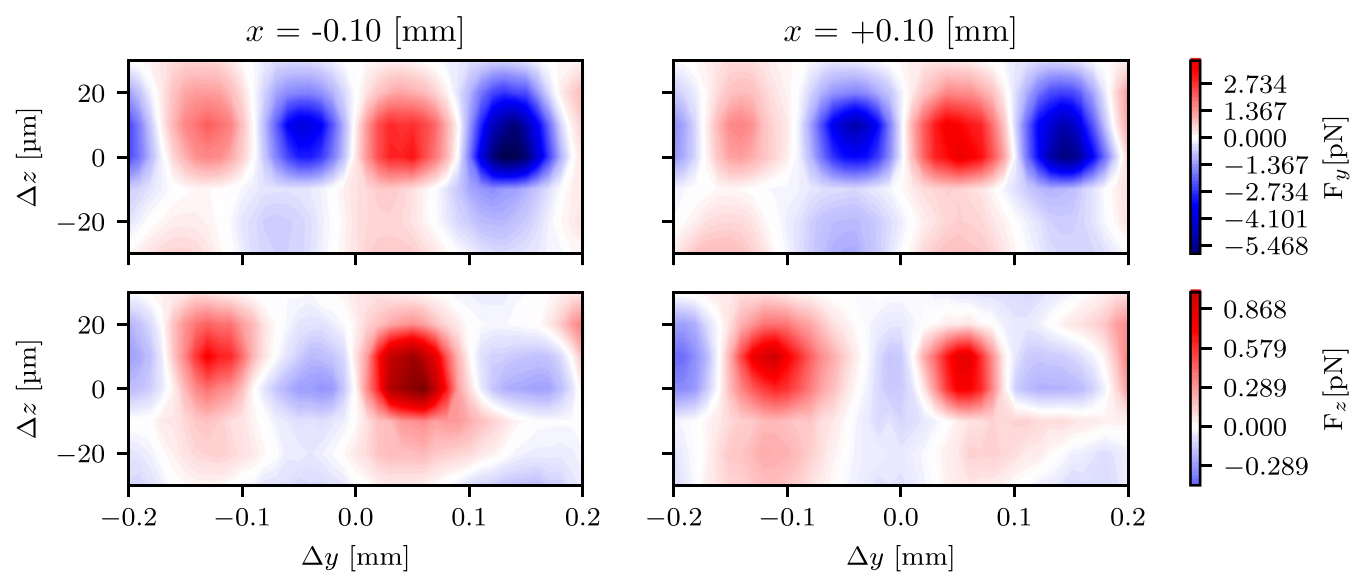

FIG. 5. Measured steady-state acoustic forces for a $4.39 \mu \mathrm{m}$ particle with $f_{\mathrm{ex}}=4.015 \mathrm{MHz}$ and $V_{\mathrm{pp}}=10.7 \mathrm{~V}$. The top row depicts the forces along $\boldsymbol{e}_{y}$ and the bottom along $\boldsymbol{e}_{z}$. The two columns correspond to two different measurement $y z$ planes at $x=-0.1 \mathrm{~mm}$ and $x=0.1 \mathrm{~mm}$, respectively.

for both measurements. More details regarding the protocol of those measurements can be found in [37] by Lamprecht $e t a l$.

We defined two $y z$ measurement planes, with $x_{1}=$ $-0.1 \mathrm{~mm}$ and $x_{2}=0.1 \mathrm{~mm}$, respectively. In each plane we defined a grid in $y_{i} \in\{-0.20,0.19, \ldots, 0.20\} \mathrm{mm}$ and $z_{j} \in$ $\{-30,-20, \ldots, 30\} \mu \mathrm{m}$. At each point $\left(y_{i}, z_{j}\right)$ we measured the forces in all three dimensions five times for $3 \mathrm{~s}$ each. Our excitation frequency was set to $f_{\mathrm{ex}}=4.015 \mathrm{MHz}$ and the applied voltage was $U_{\mathrm{pp}}=10.7 \mathrm{~V}$. We choose $f_{\mathrm{ex}}$ based on a frequency sweep and the corresponding maximal forces in this sweep. With the chosen $f_{\text {ex }}$ and the fluid speed of sound $c_{\mathrm{f}} \approx 1500 \mathrm{~m} \mathrm{~s}^{-1}$, we obtain the theoretical acoustic wavelength of $\lambda_{\mathrm{p}}=\frac{c_{\mathrm{f}}}{f_{\mathrm{ex}}} \approx 375 \mu \mathrm{m}$. Hence, with the frequency $f_{\text {ex }}$ and a channel width of $W=3 \mathrm{~mm}, 16$ pressure nodal lines are present. For each spatial position we averaged the forces over the $3 \mathrm{~s}$ time span and also over the five repetitions.

Figures 5 and 6 visualize stationary force measurement results as contour plots for the two particle sizes. In addition,
Fig. 7 depicts the measured forces in $\boldsymbol{e}_{y}$ and $\boldsymbol{e}_{z}$ directions, when the data is additionally averaged over the seven different heights $\Delta z$. For Figs. 7(a) and 7(b), the left vertical axis is the scale for the $4.39 \mu \mathrm{m}$ particle and the right vertical axis for $2.06 \mu \mathrm{m}$ particles.

In Fig. 7(a) the force wavelength $\lambda_{\mathrm{F}}$ is estimated to be $180 \mu \mathrm{m}$, which is in line with the theoretical wavelength $\lambda_{\mathrm{F}}=\frac{\lambda_{\mathrm{p}}}{2}$. One can also note that the shape of two force measurements is consistent. The ratio of the mean maximal force amplitudes $\frac{1.25}{0.17}=7.13$ is about the same as the ratio of the cubed diameter

$$
\left(\frac{4.39 \mu \mathrm{m}}{2.06 \mu \mathrm{m}}\right)^{3} \approx 2.13^{3} \approx 9.68
$$

Based on the theoretical scaling laws we conclude that the forces in the $\boldsymbol{e}_{y}$ direction are ARF dominant.

In Fig. 7(b) one can see the measured forces in $\boldsymbol{e}_{z}$ for both particle sizes and both measurement $y z$ planes. As for the
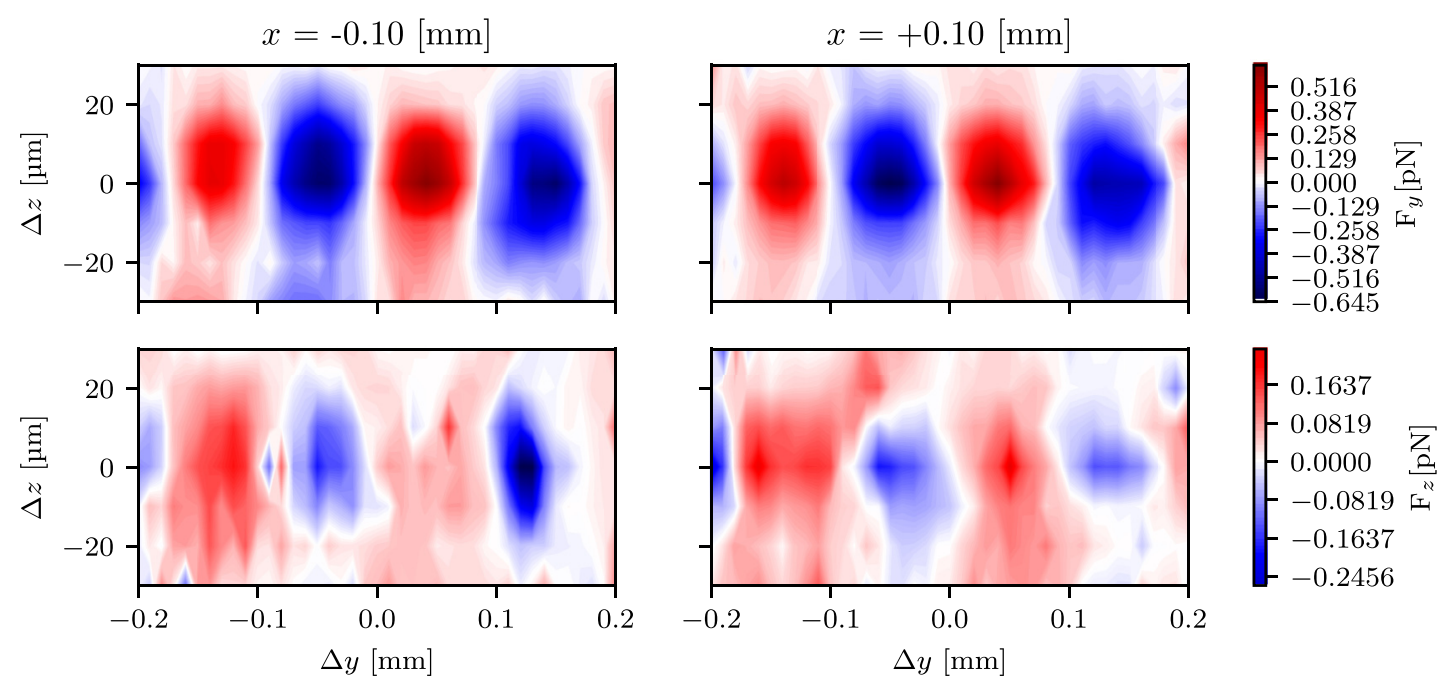

FIG. 6. Measured steady-state acoustic forces for a $2.06 \mu \mathrm{m}$ particle with $f_{\mathrm{ex}}=4.015 \mathrm{MHz}$ and $V_{\mathrm{pp}}=10.7 \mathrm{~V}$. The top row depicts the forces along $\boldsymbol{e}_{y}$ and the bottom along $\boldsymbol{e}_{z}$. The two columns correspond to two different measurement $y z$ planes at $x=0.1 \mathrm{~mm}$ and $x=0.1 \mathrm{~mm}$, respectively. 
(a)

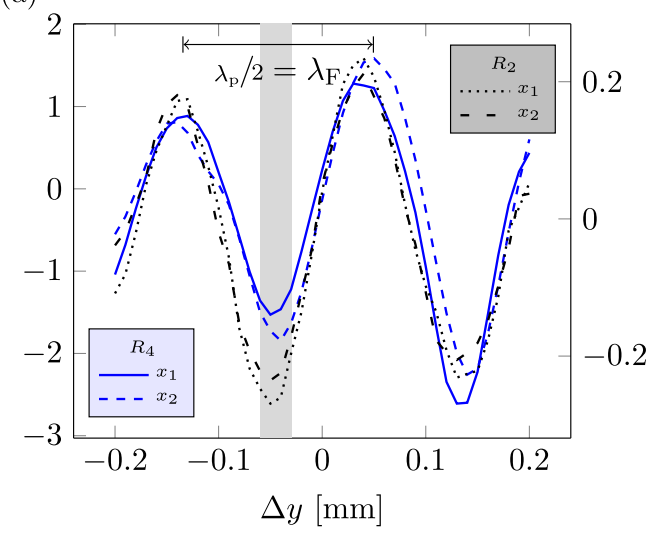

(b)

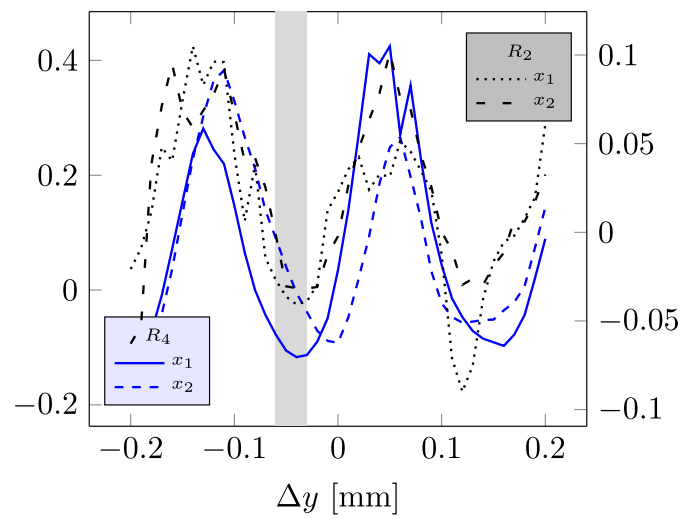

FIG. 7. Measured steady-state acoustic forces when averaged over the cavity height. All values are in pN. For each plot the left $y$ axis is the measured force on the $4.39 \mu \mathrm{m}\left(D_{4}\right)$ particle and the right one for the $2.06 \mu \mathrm{m}\left(D_{2}\right)$ particle, respectively. The gray shaded area corresponds to the positions where the time evolution is measured. (a) $F_{y}(\mathrm{pN})$. (b) $F_{z}(\mathrm{pN})$.

forces in $\boldsymbol{e}_{y}$ direction, in Fig. 7(a), the forces in $\boldsymbol{e}_{z}$ direction are averaged over all $\Delta z$. The force magnitude for both sizes is smaller than in $\boldsymbol{e}_{y}$ direction for both particle sizes. The shapes, however, are similar but not as consistent as in Fig. 7(a). The ratio of the mean maximal force amplitudes $\frac{0.25}{0.08} \approx 3.1$ is about the same as the ratio of the two diameters, which suggests that in the $\boldsymbol{e}_{z}$ direction the forces on the particle are AS dominated [see Eq. (9)].

\section{B. Measurement protocol for time evolution}

Based on a set of proof-of-concept experiments (data not shown here) and the information from numerical simulations that the AS field in a real device can substantially differ from the AS field of fluid cavity-only structure, we selected $x=0, y_{i} \in\{-0.15,-0.14, \ldots, 0.10\} \mathrm{mm}$, and $z_{j} \in$ $\{-10,0,10\} \mu \mathrm{m}$. This choice means that we measure at the same $y_{i}$ and $z_{j}$ as for the stationary force measurement. We have the same excitation frequency $\left(f_{\text {ex }}=4.015 \mathrm{MHz}\right)$ as in the stationary force measurements from before. However, we set the excitation amplitude slightly higher to $U_{\mathrm{pp}}=11.7 \mathrm{~V}$ in order to increase the signal to noise ratio (SNR).

We control the whole measuring routine with a self-written Python program. Before each measurement, the offset of the QPDs is checked and, if needed, adjusted. First we measure without US and then we measure with US on. We repeat this procedure 50 times before moving to the next location.

For the time evolution measurement, we acquire with a sampling rate of $f_{\mathrm{s}}=1.25 \mathrm{MHz}(\Delta t=0.8 \mu \mathrm{s})$ for $125 \mathrm{~ms}$ the three QPD signals, the signal for the shutter, and the dc signal for the laser as soon as the shutter starts opening $(t=-25 \mathrm{~ms}$ in Fig. 4). Between $t=0 \mathrm{~ms}$ and $t=30 \mathrm{~ms}$ the shutter is completely open and the US is switched on. Extending the measurement time further has no benefit because the particle will be outside the linear regimes of the QPDs and might move too far from the OT trapping region such that it cannot be recaptured after the laser changes to its high power state again.

We repeat 50 times per position because the particle starts sedimenting as soon as the laser power drops to the lower value. During this movement the particle still undergoes Brownian motion. Hence the trajectory is not straight along the $\boldsymbol{e}_{z}$ direction. With 50 data sets, we can average this random movement out.

Taking the approximation of Eq. (6) into account, a $2.06 \mu \mathrm{m}$ large $\mathrm{SiO}_{2}$ sphere sedimenting in water reaches its terminal velocity almost instantaneously, because the inertia term is small; additionally, the sphere travels about $0.12 R_{2}$ in $55 \mathrm{~ms}$. Therefore, after $25 \mathrm{~ms}$ the particle is still in the linear regime of the QPDz. The static gravitational force $(\tilde{m} g)$ with the added buoyancy of water is less than $40 \mathrm{fN}$ for the $2.06 \mu \mathrm{m}$ particle. This is more than six times smaller than the maximal measured force in $\boldsymbol{e}_{z}$ direction. Therefore, we assume in areas of maximal forces along $\boldsymbol{e}_{z}$ that the driving force of this movement is either the acoustic field or $F_{\mathrm{AS}}$. With an ideal sedimentation in the first $25 \mathrm{~ms}$ along $\boldsymbol{e}_{z}$, the laser spot on QPDxy does not change at all during the sedimentation.

\section{Data processing}

The acquired data is postprocessed with Python. We look at discrete points every $t_{k}=k \times 0.1 \mathrm{~ms}$ with $k \in \mathbb{N}$. In addition, we use a moving average for the data at $t_{k}$ with a centered window size of 101 data points, corresponding to a time span of $80 \mu \mathrm{s}$. Next, we subtract the data series without US from the series with US to obtain the delta voltage $\Delta V_{m}$, with $m$ being $y$ or $z$. This quantity allows us to further reduce unwanted noise. This step serves also as a data quality check because all measurements have the same protocol until $t=0 \mathrm{~ms}$. Hence the delta voltage $\Delta V_{m}$ must be zero for $t \leqslant 0 \mathrm{~ms}$. Then, we average $\Delta V_{m}$ over the 50 repetitions per spatial position $y_{i}, z_{j}$. As a last step for the time evolution plots, we normalize the data by the $\max \left[\left|\Delta V_{m}(t)\right|\right]$ for $10 \mathrm{~ms}<t<30 \mathrm{~ms}$.

\section{RESULTS AND DISCUSSION}

Figure 8(a) shows the maximal averaged voltage difference $\Delta V_{y}$ for the $2.06 \mu \mathrm{m}$ particle while having the laser in the $P_{\text {measure }}$ mode. It has the same shape as the stationary force measurement in Fig. 7(a). However, the smoothness of $\Delta V_{y}$ is worse. We attribute this to the nature of the experiment, as the recorded motion of the particle is caused by two effects: one is the acoustic field and the other is the always present Brownian 
(a)

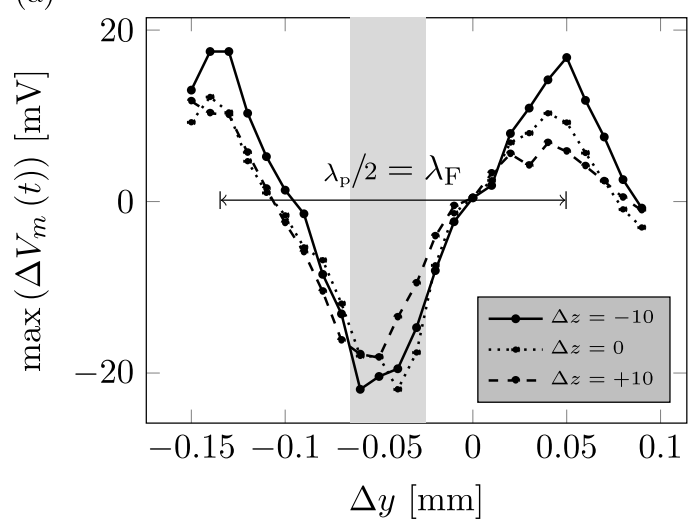

(b)

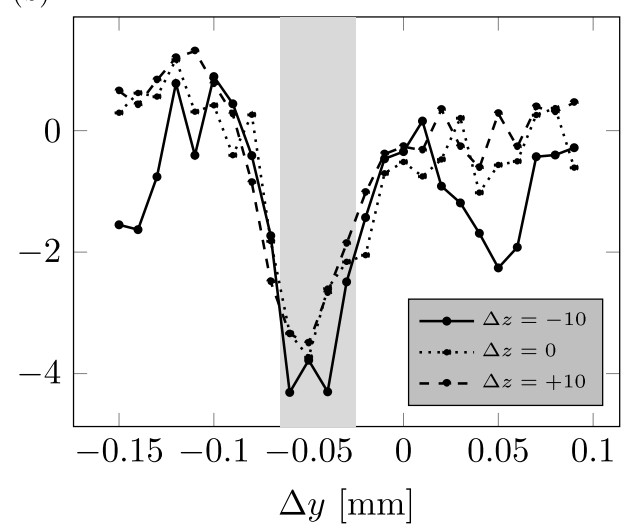

FIG. 8. Maximal $\Delta V_{y}$ and $\Delta V_{z}$ averaged over all repetitions in the time span between $35 \mathrm{~ms}$ and $55 \mathrm{~ms}$ for the three different measurement heights $\Delta z=-10,0,10 \mu \mathrm{m}$. The gray shaded area represents the $\Delta y_{i}$ of best signal strength for $\max \left[\Delta V_{z}(t)\right]$. The data points of best strength are taken for the time evolution results. The wavelength marker represents the same length as in Fig. 7(a). (a) Data for $y$ component ( $m=y$ ). (b) Data for $z$ component $(m=z)$.

motion. For the stationary force measurements the particle is fixed in place by the optical potential and the Brownian motion is negligible.

By measuring the same shape with the two experiments, we could validate our measurement protocol. As for the stationary measurements, the SNR of the evolution measurement and also shape are better for the in-plane $\boldsymbol{e}_{y}$ than the axial $\boldsymbol{e}_{z}$ [see Figs. 7(a) and 7(b)]. Nevertheless, Figs. 7(b) and 8(b) also show similar shapes. We want to stress again that the amplitudes of Fig. 8 are not comparable to each other for $\boldsymbol{e}_{y}$ and $\boldsymbol{e}_{z}$ (see Sec. III).

The numerical streaming simulations of a fluid cavity with and without the surrounding structure showed that the streaming field is a local effect in a model with surrounding structure. In our experiments we saw similar tendencies. However, not all measured spatial locations had enough actual signal strength to further investigate. In Fig. 9 we plot the time evolution of the signal for four different $\Delta y$, where it is clear that the signal is due to the acoustic field and not to noise or Brownian motion.

Since we show $\Delta V_{m}$ rather than the absolute voltage amplitudes, we can further validate our protocol. For $\frac{t}{t_{0}}<0$, where $t_{0}=\frac{1}{f_{\text {ex }}}$ and $\frac{t}{t_{0}}=0$ represents the time when the US is switched on (in Fig. $4 t=0 \mathrm{~ms}$ ), all data series in Fig. 9 are zero. All data series for $\boldsymbol{e}_{z}$ are more noisy than for $\boldsymbol{e}_{y}$. However, we also have the same amplitude of noise in $\boldsymbol{e}_{y}$ direction. But, the normalization value for the data series for $\boldsymbol{e}_{y}$ is inherently larger than for $\boldsymbol{e}_{z}$ (see Fig. 8).

For all 12 positions $\left(y_{i}, z_{j}\right)$ in Fig. 9 the signal along $\boldsymbol{e}_{y}$ starts changing as soon as the US is switched on. This is in line with the estimation of Eq. (1) for $\tau_{\mathrm{ARF}}$. For all data series $m=z$ it takes significantly more time until the movement with constant velocity starts. To further compare the results, we take as criteria the period $p^{*}=\frac{t^{*}}{t_{0}}$, when the normalized $\Delta V_{m} \geqslant 0.5$ is reached. In Table IV, the absolute periods for this criteria and the offset between the movement along $\boldsymbol{e}_{y}$ $(m=z)$ and the movement along $\boldsymbol{e}_{z}(m=y)$ are shown. Taking a different criteria value (e.g., the normalized $\Delta V_{m} \geqslant 0.3$ ) changes the absolute magnitude of the values $p^{*}$; however, the offset does not change significantly. The average for all $\Delta p^{*}$ is about 17500 which equates to $\approx 4.35 \mathrm{~ms}$ for the excitation frequency $f_{\mathrm{ex}}=4.015 \mathrm{MHz}$.

In addition, all slopes for the $y$ movement $(m=y)$ are linear almost immediately after the US is switched on. This suggests that the ARF is constant and accelerates the particle fast to its terminal velocity. The measured voltages and also their differences are linearly related to the traveled distances. Hence a constant increase in voltage, which means a constant voltage increase per time $\frac{d \Delta V_{m}}{d t}=$ const, implies a constant particle speed along the $\boldsymbol{e}_{y}$ direction. The particle trajectory in $\boldsymbol{e}_{z}$ direction is predominantly affected by the streaming field. This fluid motion takes more time until it is established. With the same reasoning as before, a linear slope for the $z$ movement $(m=z)$ in Fig. 9 implies a constant force and constant particle speed. A constant speed means a nonchanging streaming field and therefore a constant streaming velocity.

\section{CONCLUSION}

In this work we presented the measurement of the temporal evolution of the AS field and the ARF in a BAW device utilizing an OT. We slightly modified our validated optical trapping setup $[37,39]$ to accommodate the requirements of this experiment. With a temporal resolution of $\Delta t=0.8 \mu \mathrm{s}$ we could measure at least every fourth time period of excitation. We validated our measurement protocol against the stationary force field.

We monitored the trajectory of a $2.06 \mu \mathrm{m} \mathrm{SiO}_{2}$ particle as soon as the US excitation of the device started. We selected measurement positions in a standing pressure wave mode where ARF dominates in one direction and AS orthogonal to it. In addition, we chose the spatial location within the mode to maximize the amplitude of both effects. Our measurements show that the ARF is established almost immediately after the US is switched on, whereas the AS takes on average 17500 excitation periods $(4.4 \mathrm{~ms})$ longer to evolve. This time is about four times larger than the theoretical approximation with the momentum diffusion time. 


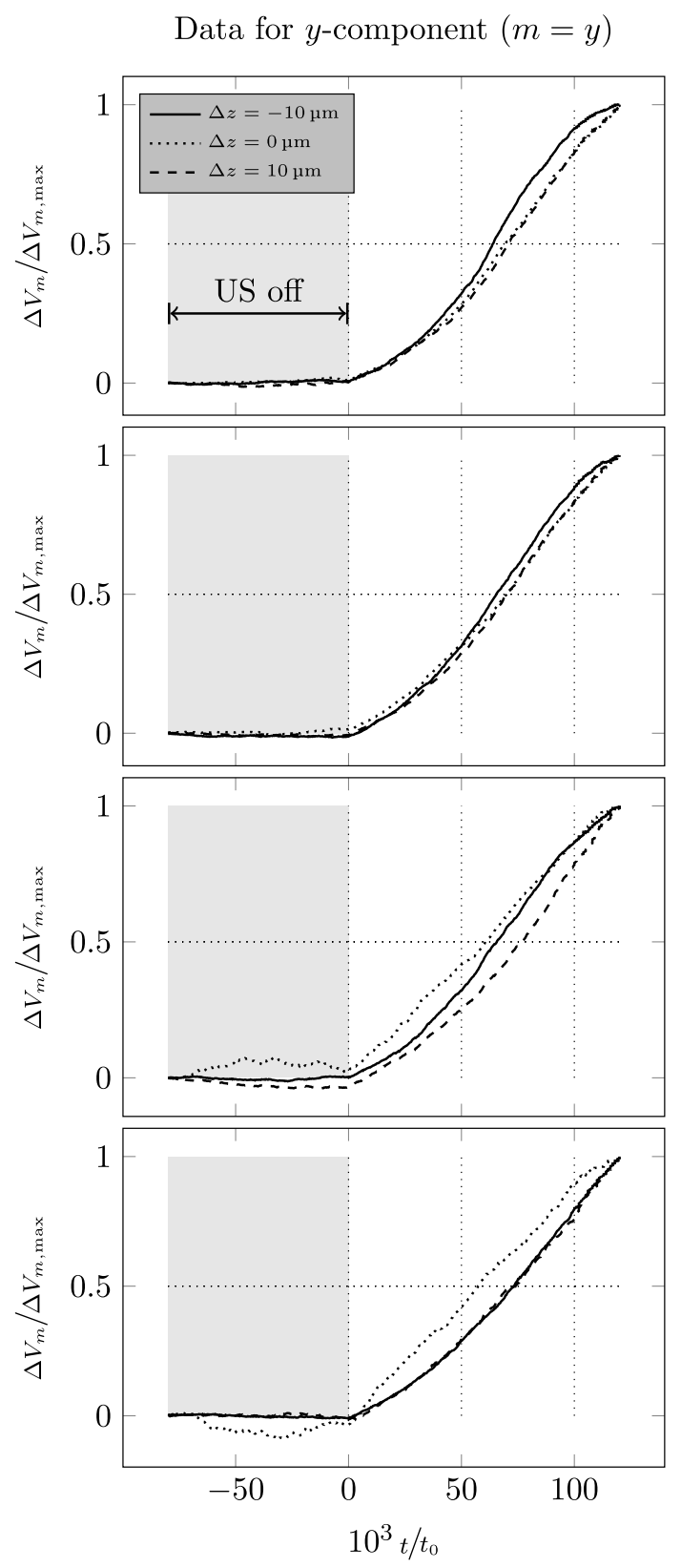

Data for $z$-component $(m=z)$
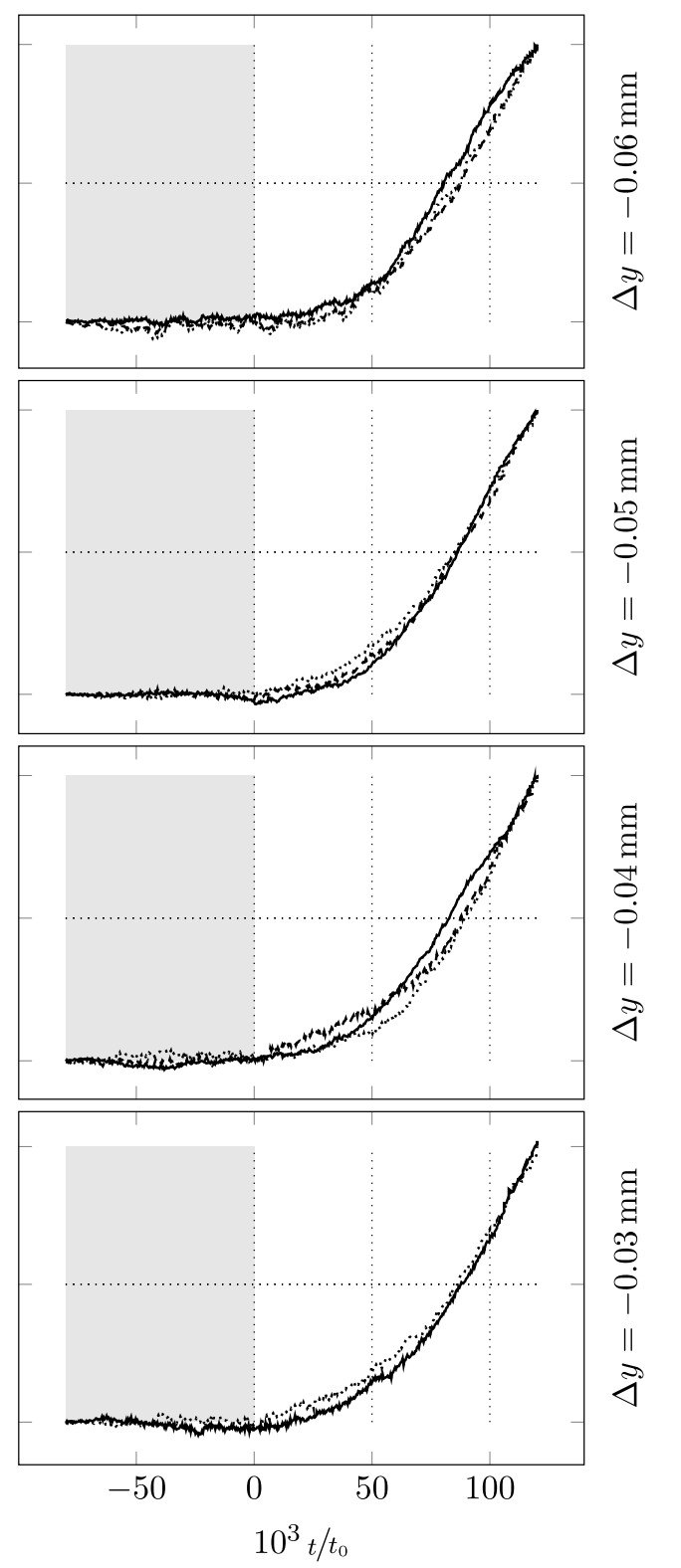

FIG. 9. Time evolution of the normalized $\Delta V_{y}$ (left column) and $\Delta V_{z}$ (right column) for the three measurement heights $\Delta z=-10,0,10 \mu \mathrm{m}$ and the positions for $\Delta y=-0.06,-0.05,-0.04,-0.03 \mathrm{~mm}$. The gray shaded area of each plot marks the time when the US is off; $t_{0}=\frac{1}{f_{\mathrm{ex}}}$.

These results show that the buildup of AS takes significantly longer than the buildup of the ARF. This temporal difference can explain why a pulsed acoustic excitation

can prevent streaming as it has been experimentally shown by Hoyos and Castro [35,36]. In addition, the results of the streaming simulations of a cavity-only model and a

TABLE IV. Absolute periods $p_{m}^{*}$ when the normalized $\Delta V_{m}>0.5$. The three values per column correspond to the three heights $\Delta z=-10,0,10 \mu \mathrm{m}$ per $\Delta y$, respectively. For $\Delta y=-0.03 \mathrm{~mm}$ and $\Delta z=10 \mu \mathrm{m}$ no data is available for $p_{z}^{*}$. The last row states the offset $\Delta p^{*}=p_{z}^{*}-p_{y}^{*}$

\begin{tabular}{lccccc}
\hline \hline$\Delta y$ & $(\mathrm{~mm})$ & -0.06 & -0.05 & -0.04 & -0.03 \\
\hline$p_{y}^{*}$ & $(\times 1000)(-)$ & $64.2,69.5,70.7$ & $65.8,70.3,70.3$ & $65.8,60.6,76.7$ & $73.5,57.4,74.3$ \\
$p_{z}^{*}$ & $(\times 1000)(-)$ & $80.7,87.9,88.3$ & $86.3,85.5,86.7$ & $83.1,89.5,87.5$ & $87.9,86.7$ \\
$\Delta p^{*}$ & $(\times 1000)(-)$ & $16.5,18.4,17.6$ & $20.5,15.2,16.4$ & $17.3,18.9,10.8$ & $14.4,29.3$ \\
\hline \hline
\end{tabular}


whole-device model show that simplified models are enough for simulations of the pressure fields; however, they cannot reflect real streaming patterns. This insight might also explain why Muller and Bruus could not reproduce the suppression of AS with a pulsed excitation in their cavity-only model.
[1] M. Antfolk, C. Magnusson, P. Augustsson, H. Lilja, and T. Laurell, Anal. Chem. 87, 9322 (2015).

[2] A. Abdulla, T. Zhang, K. Z. Ahmad, S. Li, J. Lou, and X. Ding, Anal. Chem. 92, 16170 (2020).

[3] C. Zielke, C. W. Pan, A. J. Gutierrez Ramirez, C. Feit, C. Dobson, C. Davidson, B. Sandel, and P. Abbyad, Anal. Chem. 92, 6949 (2020).

[4] M. M. Binkley, M. Cui, M. Y. Berezin, and J. M. Meacham, ACS Biomater. Sci. Eng. 6, 6108 (2020).

[5] H. Cai, Z. Ao, L. Hu, Y. Moon, Z. Wu, H.-C. Lu, J. Kim, and F. Guo, Analyst 145, 6243 (2020).

[6] N. Bose, X. Zhang, T. K. Maiti, and S. Chakraborty, Biomicrofluidics 9, 052609 (2015).

[7] Z. Wu, H. Cai, Z. Ao, A. Nunez, H. Liu, M. Bondesson, S. Guo, and F. Guo, Anal. Chem. 91, 7097 (2019).

[8] M. S. Gerlt, D. Haidas, A. Ratschat, P. Suter, P. S. Dittrich, and J. Dual, Biomicrofluidics 14, 064112 (2020).

[9] Y. Xie, H. Bachman, and T. J. Huang, TrAC, Trends Anal. Chem. 117, 280 (2019).

[10] P.-H. Huang, N. Nama, Z. Mao, P. Li, J. Rufo, Y. Chen, Y. Xie, C.-H. Wei, L. Wang, and T. J. Huang, Lab Chip 14, 4319 (2014).

[11] Y. Lin, Y. Gao, M. Wu, R. Zhou, D. Chung, G. Caraveo, and J. Xu, Lab Chip 19, 3045 (2019).

[12] A. Ozcelik and Z. Aslan, Microfluid. Nanofluid. 25, 5 (2021).

[13] A. A. Doinikov, Proc. R. Soc. Lond. 447, 447 (1931).

[14] T. Hasegawa and K. Yosioka, J. Acoust. Soc. Am. 46, 1139 (1969).

[15] K. Yosioka and Y. Kawasima, Acta Acust. Acust. 5, 167 (1955).

[16] L. P. Gor'kov, Sov. Phys. Dokl. 6, 773 (1962).

[17] H. Bruus, Lab Chip 12, 1014 (2012).

[18] W. L. M. Nyborg, Phys. Acoust. 2, 265 (1965).

[19] J. Kolb and W. L. Nyborg, J. Acoust. Soc. Am. 28, 1237 (1956).

[20] W. L. Nyborg, J. Acoust. Soc. Am. 25, 68 (1953).

[21] C. Eckart, Phys. Rev. 73, 68 (1948).

[22] N. Riley, Theor. Comput. Fluid Dyn. 10, 349 (1998).

[23] H. Schlichting, Phys. Z. 33, 327 (1932).

[24] P. B. Muller and H. Bruus, Phys. Rev. E 92, 063018 (2015).

[25] H. Bruus, Lab Chip 12, 1578 (2012).

[26] A. A. Doinikov, M. S. Gerlt, A. Pavlic, and J. Dual, Microfluid. Nanofluid. 24, 32 (2020).
[27] A. A. Doinikov, M. S. Gerlt, and J. Dual, Phys. Rev. Lett. 124, 154501 (2020).

[28] I. Leibacher, P. Hahn, and J. Dual, Microfluid. Nanofluid. 19, 923 (2015).

[29] N. Nama, P.-H. Huang, T. J. Huang, and F. Costanzo, Biomicrofluidics 10, 024124 (2016).

[30] T. Baasch, A. Pavlic, and J. Dual, Phys. Rev. E 100, 061102(R) (2019).

[31] M. Antfolk, P. B. Muller, P. Augustsson, H. Bruus, and T. Laurell, Lab Chip 14, 2791 (2014).

[32] Z. Mao, P. Li, M. Wu, H. Bachman, N. Mesyngier, X. Guo, S. Liu, F. Costanzo, and T. J. Huang, ACS Nano 11, 603 (2017).

[33] N. Hao, Z. Pei, P. Liu, H. Bachman, T. D. Naquin, P. Zhang, J. Zhang, L. Shen, S. Yang, K. Yang, S. Zhao, and T. J. Huang, Small 16, 2005179 (2020).

[34] M. Wiklund, R. Green, and M. Ohlin, Lab Chip 12, 2438 (2012).

[35] M. Hoyos and A. Castro, Ultrasonics 53, 70 (2013).

[36] A. Castro and M. Hoyos, Ultrasonics 66, 166 (2016).

[37] A. Lamprecht, S. Lakämper, T. Baasch, I. A. T. Schaap, and J. Dual, Lab Chip 16, 2682 (2016).

[38] S. Lakämper, A. Lamprecht, I. A. T. Schaap, and J. Dual, Lab Chip 15, 290 (2015).

[39] A. Lamprecht, C. Goering, I. A. T. Schaap, and J. Dual, J. Micromech. Microeng. 31, 034004 (2021).

[40] H. Bruus, in Microscale Acoustofluidics (The Royal Society of Chemistry, London, 2015), pp. 1-28.

[41] A. Lamprecht, Optical traps for characterising acoustically induced forces and torques acting on microparticles, Ph.D. thesis, ETH Zurich, 2017.

[42] See Supplemental Material at http://link.aps.org/supplemental/ 10.1103/PhysRevE.104.025104 for the two COMSOL Multiphysics 5.6 models of the whole device and cavity only. With those models the streaming fields of Fig. 2 are computed.

[43] J. K. Dreyer, K. Berg-Sørensen, and L. Oddershede, Appl. Opt. 43, 1991 (2004).

[44] R. Barnkob, P. Augustsson, T. Laurell, and H. Bruus, Phys. Rev. E 86, 056307 (2012).

[45] M. Settnes and H. Bruus, Phys. Rev. E 85, 016327 (2012). 